\title{
Prix et distinctions
}

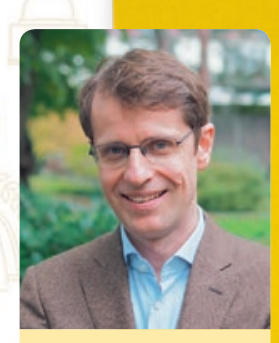

Prof. Dr. Botond Roska (@ IOB.ch).

\section{Greenberg Prize}

Prof. Dr. Botond Roska erhält den Sanford and Susan Greenberg Visionary Prize «für einzigartig wertvolle Forschung mit den grössten Auswirkungen auf den Fortschritt bei der Wiederherstellung des menschlichen Augenlichts». Prof. Dr. Roska ist Direktor am Institut für Molekulare und Klinische Ophthalmologie Basel (IOB) und Professor an der medizinischen und der naturwissenschaftlichen Fakultät der Universität Basel. Ziel des GreenbergPreises ist es, eine weltweite Forschungsgemeinschaft zu schaffen, die mit ihren kollektiven Fähigkeiten und Ressourcen zur Beendigung der Blindheit beiträgt. Die Preisverleihung wird in Washington D.C. am 14. Dezember stattfinden. Das Preisgeld von USD 250000 ist für die weitere Forschung bestimmt.

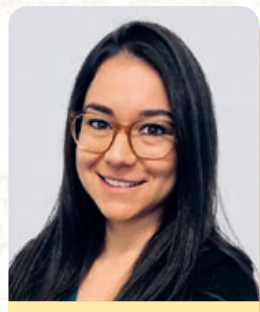

Dr. Joanna Triscott (c zvg/Courtesy of Joanna Triscott).

\section{Johanna Dürmüller-Bol DBMR Forschungspreis}

Dr. Joanna Triscott wird mit dem diesjährigen Johanna Dürmüller-Bol DBMR

Forschungspreis (DMBR: Department for BioMedical Research) der Universität Bern ausgezeichnet. Dr. Triscott wird für ihre Forschung zu Stoffwechselprozessen von Prostatakrebstumoren ausgezeichnet. Der mit CHF 30000 dotierte Nachwuchs-Forschungspreis wurde am 4. November, am «Day of BioMedical Research», verliehen.

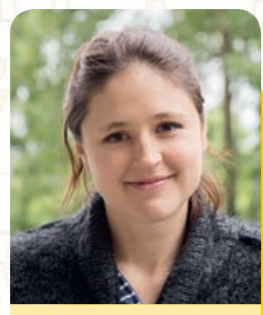

Dr. Dasha Nelidova (๔ IOB.ch).

\section{Vier Preise für Dasha Nelidova}

Dr. Dasha Nelidova, Postdoc am Institut für Molekulare und Klinische Ophthalmologie Basel (IOB), gewann einen der wichtigsten Forschungspreise für junge Wissenschaftler: den Science \& SciLifeLab Prize for Young Scientists. Der internationale Preis wird für die besten und relevantesten Doktorarbeiten verliehen.

Dr. Nelidova gewinnt den Preis in der Kategorie Molekulare Medizin, für die Entwicklung einer optogenetischen Technologie, die das Potenzial hat, fast vollständig Erblindeten wieder Sehwahrnehmungen im nahen Infrarotbereich zu ermöglichen. Der Preis ist mit USD 10000 dotiert.

Es ist bereits der vierte Preis für Dr. Dasha Nelidova in diesem Jahr, nach dem Swiss OphthAward 2020 für die beste experimentelle Arbeit, dem grundlagenwissenschaftlichen Forschungspreis 2020 der PRO RETINA Deutschland e.V. und Retina Suisse und einem Ophthalmology Star Award 2020.

\section{Prix de la Fondation pour la Recherche sur le Diabète 2020}

Les Dr Julien Vaucher et Pr Aurélien Thomas (CHUV-UNIL) ont reçu le Prix de la Fondation pour la Recherche sur le Diabète 2020. Sur avis d'un jury indépendant, le comité scientifique de la Fondation a retenu leur projet qui sera soutenu à hauteur de CHF 84111. En utilisant les données de 6733 individus inclus dans une large étude épidémiologique basée au Centre hospitalier universitaire vaudois depuis 2003 , le but de ce projet est de mieux comprendre l'association entre arsenic et diabète, en s'appuyant sur une approche génétique éprouvée qui permettra d'établir, ou pas, l'effet direct de l'arsenic sur le développement du diabète.

Depuis près de 30 ans, la Fondation pour la Recherche sur le Diabète soutient chaque année un ou plusieurs projets de recherche sur le diabète menés au sein des pôles d'expertises universitaires romands.

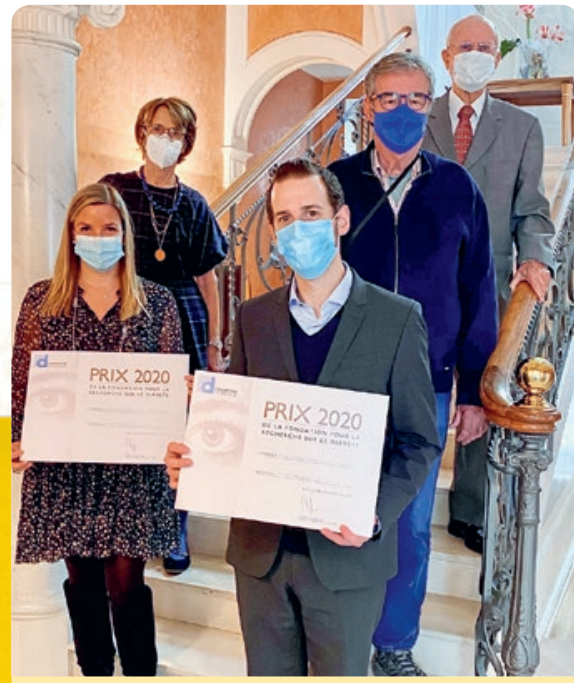

En bas à dr., Pr Aurélien Thomas, co-lauréat du Prix de la Fondation 2020, à côté de Victoria Chmielewski, directrice de la Fondation. En haut de g. à dr.: Catherine Fauchier-Magnan, vice-présidente, Pierre-François Unger, président, et Michel Rossetti, membre du comité de Fondation (@ Fondation pour la Recherche sur le Diabète). 